\title{
资源互补效应对多样性-生产力关系的影响
}

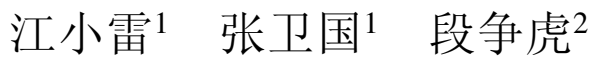 \\ （1 农业部草地农业生态系统学重点开放实验室, 兰州大学草地农业科技学院, 兰州 730020) \\ (2 中国科学院寒区早区环境与工程研究所, 兰州 730000)
}

摘 要 许多有关物种多样性-生态系统功能关系的观察、理论和实验研究都表明, 在局域尺度范围内, 植物种多 样性对生态系统生产力存在正效应。然而, 对于促成这种关系的潜在生态学机制却缺乏足够的了解。该实验利用 9 种一年生栽培牧草, 采用各物种单播及混播的方法, 构建不同多样性梯度的实验群落, 对物种多样性与生态系 统生产力的关系及资源互补效应对系统生产力的影响进行了研究。结果表明, 在一年生植物群落内, 植物种多样 性在一定程度内对系统生产力存在正效应, 物种多样性与生产力呈二次函数关系, 关系式为 $y=-98.449 x^{2}+$ $1039.2 x-42.407,\left(R^{2}=0.4231\right)$ 。各物种在资源利用、生长速度和竞争能力等功能特征方面存在较大差异, 最 高产物种和最低产物种间产量相差 5.8 倍。在同一多样性梯度内, 不同物种组合的群落间生产力和互补效应也存 在较大差异, 说明物种的成分对生态系统生产力也有重要影响。同时, 在混播群落中程度不同地存在着资源的互 补性利用, 说明物种多样性对系统生产力有增强作用, 但相关分析表明, 互补效应和物种多样性间不存在显著相 关关系。互补效应的 4 种计算方法所反映的资源互补程度有所不同, 每种方法各有利弊, 在对系统的多样性效应 作用机制进行评价时, 应根据具体情况, 同时采用几种方法, 以利于对资源互补效应做出恰当的估测。

关键词 植物种多样性 生产力 资源互补 超产效应 作用机理 物种成分

\section{EFFECTS OF COMPLEMENTARITY ON DIVERSITY-PRODUCTIVITY RELATIONSHIP}

\author{
JIANG Xiao-Lei ${ }^{1}$ ZHANG Wei-Guo ${ }^{1}$ and DUAN Zheng-Hu ${ }^{2}$ \\ (1 Key Laboratory of Grassland Agro-Ecosystem, Ministry of Agriculture, College of Pastoral Agriculture Science and Technology, \\ Lanzhou University, Lanzhou 730020, China) \\ (2 Cold \& Arid Regions Environmental and Engineering Research Institute, Chinese Academy of Sciences, Lanzhou 730000, China)
}

\begin{abstract}
A number of observational, theoretical and experimental studies have indicated that local plant species diversity can have positive effects on ecosystem productivity; however, little is known about the ecological mechanisms that regulate this relationship. To investigate the relationship between plant species diversity and ecosystem productivity and the effect of resource complementarity on productivity, we established experimental communities containing different levels of diversity using nine cultivated annual plant species. To address questions of complementary resource use, we planted each species in monocultures as well as in different polycultures, which allowed us to evaluate complementary effects. Complementarity was assessed by using four analytical methods. The first two methods were tested for an absolute increase in productivity with increasing diversity. The hypothesis was that a polyculture would outperform the most productive monoculture of a component species, defined as overyielding effect $1\left(O V_{1}\right)$, and a polyculture would perform better than the average yield of monocultures of the component species, defined as overyielding effect $2\left(\mathrm{OV}_{2}\right)$. The third method measured the relative yield of the polyculture, Relative Yield Totals ( RYTs), and the last method measured the $D$ value, the proportional deviation of the productivity of a polyculture from its expected value. The results indicated that, to a certain extent, species diversity showed a positive effect on community productivity. The relationship between species richness and community productivity could be represented by a quadratic equation $y=-98.449 x^{2}+1039.2 x-42.407\left(R^{2}=0.423\right)$. Large differences in productivity were found among treatments with similar levels of diversity indicating that species composition had an important impact on community productivity. Calculation of a complementarity index indicated that about $40 \%$ of the polycultures outperformed its most productive component monoculture, more than $95 \%$ of the polycultures performed better than the average yield of its component monocultures, and more than $50 \%$ of the polycultures had significant RYTs $>1$ and $D>0$. These results suggest that resource complementarity was partly responsible for the positive effect of species diversity on productivity. Complementarity, however, was not significantly related to
\end{abstract}


species diversity. Four analytic methods were used for estimating the net outcome of complementary effects and the different levels of ecological interaction in a community. Each method had its advantages and disadvantages. Therefore, in assessing how complementarity influences ecosystem productivity, different methods should be integrated.

Key words Plant species diversity, Productivity, Resource complementarity, Overyielding, Mechanism, Species composition

在多样性-生态系统功能关系研究中, 许多人工 系统、半人工系统及天然草地植物群落的研究都表 明, 物种多样性对生态系统功能, 特别是系统生产力 有重要影响 (Tilman et al., 1996; Naeem et al., 1996; Symstad et al., 1998; Hector et al., 1999; Fridley, 2003), 然而, 对于促成这种关系的潜在生态学 机制却缺乏足够的了解。物种多样性可通过各种机 制对生态系统功能产生影响（Wardle, 1999; Chapin III et al., 1993; Loreau, 2000; Tilman, 2000; Fridley, 2001), 探讨特定系统中控制多样性-生产力关系的 可能作用机制并对其进行定量描述, 对于正确理解 物种多样性变化的生态学意义非常重要。一般而 言, 资源互补 (Resource complementarity) 是目前解释 物种多样性对生态系统生产力产生正效应涉及最 多、且广为接受的机制之一 ( Jonsson \& Malmqvist, 2003)。资源互补 (或生态位互补) 理论认为, 多样性 的增加使群落中物种功能特性 (如根系深度、冠层高 度、生长速度、竞争能力及对不良环境的耐受力等） 的多样化增加, 从而可实现对有限的资源在不同的 时间、空间, 以不同的方式进行利用, 使资源利用率 最大化, 进而导致系统功能水平的提高 (Tilman et al .,1997; Hooper, 1998; Loreau, 1998a)。例如, 深根 系物种和浅根系物种组合, 可最大限度地利用不同 土层的水分; 不同冠层结构的物种搭配, 可有效利用 空间资源和光能资源; 不同物候期的物种构建的群 落可在不同阶段充分利用有限的养分。同时, 不同 的物种对各种资源的利用程度及同种资源的不同形 式的偏爱亦有所差异, 因此, 多样性程度较高的群落 可实现资源利用的互补, 减少系统中资源的浪费, 使 群落生产力达到较高的水平 (Hooper \& Vitousek, 1997)。有关草地生态系统植物多样性和互补效应 对生态系统功能影响的研究尚不多见 (Hooper, 1998; Dukes, 2001; Loreau \& Hector, 2001; Fridley, 2003; Hooper \& Dukes, 2004), 且各研究在方法、内容 及目的等方面各有千秋, 并未就多样性、互补效应对 生态系统功能的作用得出规律性结论, 因此, 关于多 样性-生态系统功能关系的作用机制问题仍需进行 深入的研究和探讨。据此, 我们在改进他人的研究
方法的基础上, 利用人工构建的多样性植物群落就 资源互补效应对多样性-生产力关系的影响进行了 研究。在群落的建植上采用各物种单播和不同多样 性梯度组合混播的方法, 为互补效应的准确测定提 供了有效途径。同时采用几种不同的互补效应计算 方法, 比较各种统计变量所反映的互补效应对系统 功能的影响程度, 以期为多样性-生态系统功能关系 研究提供有价值的参考依据。

\section{1 材料和方法}

\section{1 实验地概况}

实验设在甘肃省景泰县引黄一期灌区, 地理位 置 $107^{\circ} 03^{\prime} \mathrm{N}, 37^{\circ} 11^{\prime} \mathrm{E}$, 海拔 $1630 \mathrm{~m}$ 。年平均气温 $8.2{ }^{\circ} \mathrm{C}$, 无霜期 $141 \mathrm{~d}$, 总太阳辐射量为 $6001 \mathrm{M} \mathrm{J} \cdot$ $\mathrm{M}^{-2} \cdot \mathrm{a}^{-1}, \geqslant 0{ }^{\circ} \mathrm{C}$ 的活动积温 $3614.8{ }^{\circ} \mathrm{C}, \geqslant 10{ }^{\circ} \mathrm{C}$ 的活动积温 $3038.2{ }^{\circ} \mathrm{C}$, 年平均降水量 $182 \mathrm{~mm}$, 年蒸 发量 $2000 \mathrm{~mm}$ 。土壤为荒漠灰钻土, 土壤 $\mathrm{pH}$ 值 8.5 。 实验地为耕作多年的农田, 具有较一致的养分条件, 灌溉条件良好。

\section{2 材料和方法}

供试材料为 9 种一年生栽培牧草: 燕麦( Avena sativa)、多花黑麦草 ( Lolium multiflorum) 、湖南稷子 ( Echinochloa crusgalli var. frumentacea)、苏丹草 ( Sorghum sudanense)、高丹草( Sorghum vulgare $\times$ S. sudanense)、白花草木樨 (Melilotus alba)、箭口踠豆 (Vicia sativa)、毛苔子 ( Vicia villosa)、籽粒苋 (Amaranthus hypochondriacus)。草种由甘肃创绿草业公司提供。

实验采用完全随机区组设计,共设 9 个多样性 水平, 21 个处理 (9 个种单播; $2 \sim 5$ 个种各 2 种组合; $6 \sim 9$ 个种各 1 种组合), 3 组重复, 共 63 个小区。小 区面积 $3 \mathrm{~m} \times 4 \mathrm{~m}$, 由 $0.5 \mathrm{~m}$ 保护行隔离。各处理所 含物种由 9 种植物组成的物种库中随机抽取产生。 每个种在各处理中的播量按下式计算:

种 $i$ 的实际播量 $=$ 种 $i$ 的理论播量 / (种 $i$ 的种 用价值 $\times$ 种 $i$ 所在处理的物种数)

各物种均于 2002 年 4 月初人工条播, 实验地建 植后采用常规法进行田间管理。

用地上生物量代替群落生产力 ( Fridley, 2003)。 
在每小区内设 $50 \mathrm{~cm} \times 50 \mathrm{~cm}$ 样方 3 个, 取样后混 合,再取其 $1 / 3$ 用作各项指标的测定。分别于 7 月 中旬和 9 月中旬两次用刈割法测定样方内各物种的 高度、盖度、密度和生物量, 以两次生物量烘干重之 和作为各群落生产力水平的度量指标。

\section{3 互补效应的计算方法}

选择 4 种指数检验混播群落中的生态位互补效应。

1) 超产效应 1（Transgressive overyielding effect, $\left.O V_{1}\right)$ : 混播群落生产力超过该群落组分种中生产力 最高物种的单产 (Hector，1998)，计算公式为：

$O V_{1}=\left(B_{\text {poly }}-\max B_{i \text { mono }}\right) / \max B_{i \text { mono }}$

式中: $B_{\text {poly }}$ 为混播群落产量, $\max B_{i \text { mono }}$ 为混播群落组 分种中最高产物种的单产。 $O V_{1}>0$, 表明存在超 产效应。

2) 超产效应 2( $\left.\mathrm{OV}_{2}\right)$ : 混播群落的生产力超过 该群落内各组分种的平均单产 (Tilman, 1999), 计 算公式为

$O V_{2}=\left(B_{\text {poly }}-\right.$ mean $\left.B_{\text {mono }}\right) /$ mean $B_{\text {mono }}$

式中: mean $B_{\text {mono }}$ 为混播群落中各组分种的平均单 产。 $O V_{2}>0$, 表明存在超产效应。

3) 相对产量总和 (Relative yield totals, RYTs) (Hooper，1998）: 其计算公式为:

$$
\begin{aligned}
& R Y_{i}=Y_{i \text { poly }} /\left(Y_{i \text { mono }} / n\right) \\
& R Y T=\sum R Y_{i} / n \\
& R Y T s=\text { mean } R Y T_{j}
\end{aligned}
$$

式中: $R Y_{i}$ 为种 $i$ 在混播群落中的相对产量, $Y_{i \mathrm{poly}}$ 为 种 $i$ 在混播群落中的产量, $Y_{i \text { mono }}$ 为种 $i$ 的单播产 量, $n$ 为混播群落的物种数, $R Y T$ 为混播群落的相对 产量总和, RYTs 为各处理的平均值。

对一特定的混播群落而言, 某一种的相对产量 $(R Y)$ 大于 1 , 说明该种在混播群落中的表现优于单 播; 相对产量等于 1 , 说明该种在混播群落中的表现 与单播的相同; 相对产量小于 1 , 则表明该种在混播 群落中的生长受种间竞争的限制甚于种内竞争的限 制。RYTs > 1, 表明群落中存在资源互补性利用 (或种间促进作用)(Dukes，2001); RYTs = 1，表明 混播群落种间的竞争作用与单播群落的种内竞争作 用相同, 或混播群落内某些种较高的相对产量被另 一些种较低的相对产量所抵消; RYTs $<1$, 表明群 落中种间竞争作用大于种内竞争作用。

4） $D$ 值: 混播群落产量与期望产量之偏差比 (Loreau，1998b), 其计算方法为:

$D=$ mean $D_{j}$

$$
D_{j}=\Sigma\left(O_{i}-E_{i}\right) / E_{i}
$$

式中: $D_{j}$ 为群落 $j$ 的 $D$ 值, $O_{i}$ 为种 $i$ 在混播群落中 的实际产量, $E_{i}$ 为种 $i$ 对应的期望产量。 $D>0$ 表 明群落中存在资源互补性利用。

\section{2 结果与分析}

\section{1 物种多样性对群落生产力的影响}

由图 1 可见, 各物种生产力水平变化较大 $(p<$ $0.05)$, 表现最佳物种为苏丹草, 单播群落产量达 $3056.8 \mathrm{~g} \cdot \mathrm{m}^{-2}$, 其次为燕麦和多花黑麦草, 单播群 落产量分别为 2244.4 和 $1632.4 \mathrm{~g} \cdot \mathrm{m}^{-2}$, 豆科牧草 表现较差, 其生产水平在 $518.8 \sim 901.6 \mathrm{~g} \bullet \mathrm{m}^{-2}$. 最 高产物种苏丹草与最低产物种籽粒苋之间生物量相 差 5.8 倍, 说明不同物种间在养分利用、生长速率和 竞争能力等功能特征方面存在较大差异。

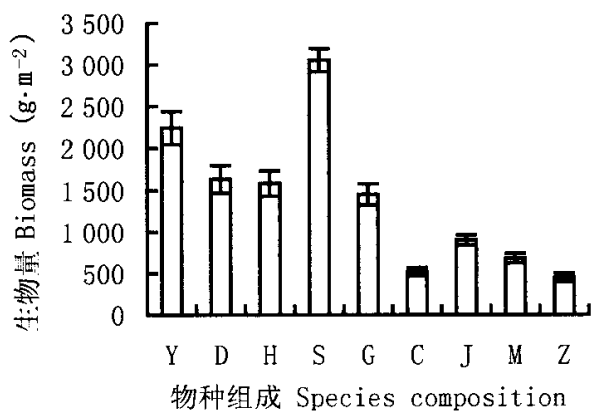

图 1 物种成分对生产力的影响

Fig. 1 Effects of species composition on productivity

$\mathrm{Y}$ : 燕麦 Avena sativa D: 多花黑麦草 Lolium multiflorum $\mathrm{H}$ : 湖南 稷子 Echinochloc crusgalli var. frumentacea S: 苏丹草 Sorghum sudanense G: 高丹草 Sorghum vulgare $\times S$. sudanense C: 草木樨 Melilotus alba J: 箭口踠豆 Vicia sativa M: 毛苦子Vicia villosa Z: 籽粒苋 $A$ maranthus hypochondriacus

图 2 表明, 各混播群落生产力变化幅度也较 大, 平均而言, 生产力水平最高的群落为燕麦 + 湖 南稷子 + 苏丹草 + 多花黑麦草 + 箭 $\square$ 碗豆 5 个种的 组合, 为 $3143.8 \mathrm{~g} \cdot \mathrm{m}^{-2}$, 生产力水平最低的群落为 高丹草 + 多花黑麦草两个种的组合, 仅为 $1610.0 \mathrm{~g}$ $\cdot \mathrm{m}^{-2}$ 。回归分析表明, 物种多样性与生产力关系呈 明显的单峰格局, 即: 生产力随物种多样性的增加呈 增加趋势, 当物种多样性达到一定程度时 (本研究中 为 5 种), 生产力达最大值, 之后随着物种多样性的 继续增加, 生产力呈下降趋势, 其关系式为: $y=$ $-98.449 x^{2}+1039.2 x-42.407\left(R^{2}=0.4231\right)$ 。 但具体分析不同的物种组合时可以发现, 当物种数 一定时, 不同物种组成的群落间其生产力存在较大 的差异 (表1), 如同为两个种的组合, 毛苕子 + 燕麦 
表 1 混播群落中各物种相对产量 $(\boldsymbol{R} Y)$ 及群落相对产量总和 $(\boldsymbol{R} Y \boldsymbol{T} \boldsymbol{s})$

Table 1 Relative yields (RY) of each species in mixtures and relative yield totals ( $R Y T s$ ) for those mixtures ( mean $\pm 1 S E, n=3$ )

\begin{tabular}{|c|c|c|c|c|c|c|c|c|c|c|c|}
\hline \multirow{2}{*}{$\begin{array}{c}\text { 处理 } \\
\text { Treatment }\end{array}$} & \multirow{2}{*}{$\begin{array}{l}\text { 种数 } \\
\text { No. of } \\
\text { species }\end{array}$} & \multicolumn{9}{|c|}{ Relative yield } & \multirow{2}{*}{ RYTs } \\
\hline & & $\mathrm{Y}$ & D & $\mathrm{H}$ & S & G & C & $\mathrm{J}$ & M & Z & \\
\hline MY & 2 & $2.26 \pm 0.24$ & & & & & & & $0.27 \pm 0.05$ & & $1.41 \pm 0.16$ \\
\hline GD & 2 & & $1.72 \pm 0.41$ & & & $0.26 \pm 0.11$ & & & & & $0.99 \pm 0.04$ \\
\hline YHS & 3 & $3.33 \pm 0.37$ & & $0.45 \pm 0.17$ & $0.51 \pm 0.10$ & & & & & & $1.43 \pm 0.13$ \\
\hline MYH & 3 & $3.13 \pm 0.37$ & & $0.57 \pm 0.13$ & & & & & $1.25 \pm 0.15$ & & $1.65 \pm 0.21$ \\
\hline YSDH & 4 & $3.89 \pm 0.48$ & $1.31 \pm 0.14$ & $0.45 \pm 0.08$ & $0.15 \pm 0.02$ & & & & & & $1.45 \pm 0.05$ \\
\hline ZGJH & 4 & & & $1.92 \pm 0.18$ & & $2.63 \pm 0.24$ & & $0.66 \pm 0.17$ & & $0.88 \pm 0.19$ & $1.52 \pm 0.13$ \\
\hline CJYMZ & 5 & $5.79 \pm 1.73$ & & & & & $0.66 \pm 0.15$ & $0.71 \pm 0.18$ & $0.43 \pm 0.09$ & $0.86 \pm 0.19$ & $1.69 \pm 0.16$ \\
\hline ZGMSC & 5 & & & & $1.74 \pm 0.17$ & $3.58 \pm 0.38$ & $0.69 \pm 0.13$ & & $1.24 \pm 0.19$ & $0.69 \pm 0.12$ & $1.59 \pm 0.14$ \\
\hline CMHYSZ & 6 & $5.45 \pm 1.28$ & & $0.48 \pm 0.05$ & $0.39 \pm 0.04$ & & $0.87 \pm 0.19$ & & $0.59 \pm 0.11$ & $0.56 \pm 0.10$ & $1.39 \pm 0.16$ \\
\hline CJSHDYZ & 7 & $4.96 \pm 0.72$ & $0.91 \pm 0.17$ & $0.66 \pm 0.08$ & $0.23 \pm 0.06$ & & $1.22 \pm 0.16$ & $0.73 \pm 0.18$ & & $0.39 \pm 0.05$ & $1.14 \pm 0.13$ \\
\hline ZCMDHGYS & 8 & $5.34 \pm 1.31$ & $0.69 \pm 0.12$ & $0.54 \pm 0.12$ & $0.26 \pm 0.03$ & $0.40 \pm 0.13$ & $0.96 \pm 0.07$ & & $0.68 \pm 0.13$ & $0.79 \pm 0.18$ & $1.07 \pm 0.61$ \\
\hline ZCJMDHGYS & 9 & $5.21 \pm 1.09$ & $0.94 \pm 0.13$ & $0.76 \pm 0.23$ & $0.22 \pm 0.07$ & $0.51 \pm 0.11$ & $0.67 \pm 0.12$ & $0.81 \pm 0.17$ & $0.79 \pm 0.15$ & $0.31 \pm 0.07$ & $1.13 \pm 0.29$ \\
\hline
\end{tabular}

Y、D、H、S、G、C、J、M、Z: 见图 1 See Fig. 1

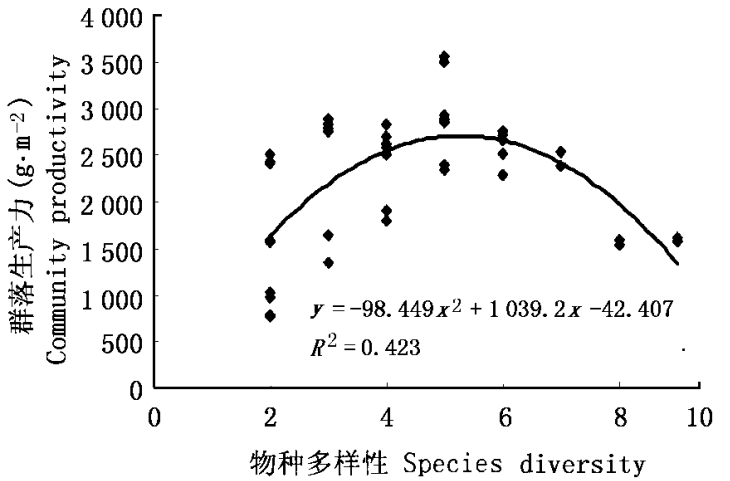

图 2 物种多样性对生产力的影响

Fig.2 Effects of species diversity on productivity

群落与高丹草 + 多花黑麦草群落生物量相差 36\%; 同样, 4 个种的组合中, 燕麦 + 苏丹草 + 多花黑麦草 + 湖南稷子群落与籽粒苋 + 多花黑麦草 + 箭口踠豆 + 湖南稷子群落生产力相差 $35 \%$ 。这说明除物种 多样性外, 物种的成分对生态系统生产力也有重要 影响。

\section{2 资源互补效应对生产力的影响}

4 种互补效应指数分析均表明, 在混播群落中 程度不同地存在着资源的互补性利用(图 3, 图 4, 表 1 , 表 2), 即物种多样性对群落生产力有增强作用。 但回归分析表明, 多样性与互补效应间不存在显著 相关性 ( $\left.p=0.7631, R^{2}=0.2204\right)$, 这说明混播 群落中物种间的相互关系较为复杂, 不单纯表现为 生态位互补效应。

超产效应 1 计算结果表明 (图 3), 在 12 个多样 性组合中, 约 $40 \%$ 的群落 $O V_{1}>0$, 即混播群落生产 力超过其生产力水平最高组分种的单播产量, 存在

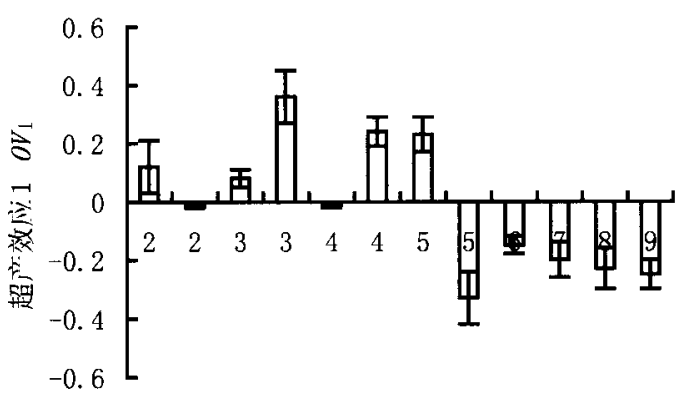

物种多样性 Species richness

图 3 物种多样性对超产效应 1 的影响

Fig.3 Effect of species diversity on overyielding effect 1

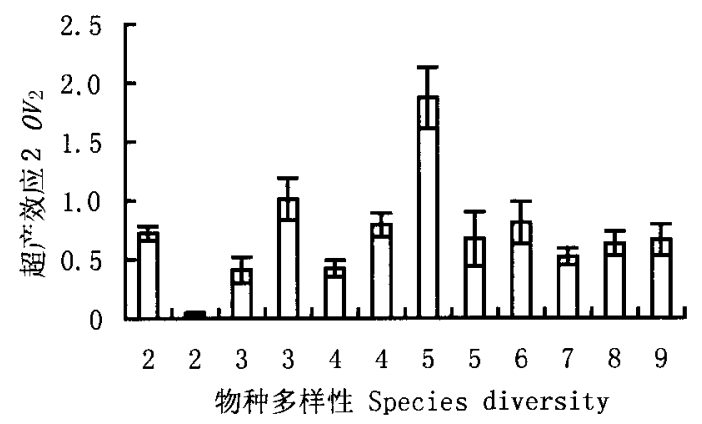

图 4 物种多样性对超产效应 2 的影响

Fig.4 Effect of species diversity on overyielding effect 2

生态位互补效应。超产幅度最大的组合为毛苕子 + 燕麦 + 湖南稷子 3 个种的群落, 超产达 $35.7 \%$; 超产 幅度最小的组合是燕麦 + 湖南稷子 + 苏丹草 3 个种 的群落, 仅超产 $8.3 \%$ 。另外约 60 个多样性较高的 群落均存在负超产效应, 即多样性对群落生产力产 生负效应。 
超产效应 2 计算结果表明 (图 4), 所有的混播 群落都程度不同的存在资源互补效应,超产幅度最 大的群落为草木樨 + 箭 $\square$ 竚豆 + 毛苕子 + 燕麦 + 籽 粒苋 5 个种的组合, 超产达 $187.6 \%$, 超产幅度最小 的群落为高丹草 + 多花黑麦草 2 个种的组合, 仅超 产 $4.3 \%$ 。由此可见, $O V_{2}$ 指数是衡量互补效应较 为温和的指标。

相对产量 (RYTs 和 $D$ 值)计算结果具有较为一 致的变化趋势 $($ 表 1 , 表 2), 除高丹草 + 多花黑麦草 群落和 7、8、9 个种的群落外 $(R Y T s=0.99,1.14$, 1.06 和 $1.13, p>0.05 ; D=0.07, p>0.05)$, 其 余各群落均表现出显著的互补效应 $(R Y T s>1, p<$ $0.05 ; D>0, p<0.05)$ 。从各组分的 $R Y T$ 和 $D$ 值
可以看出, 各物种对混作的反应差别较大, 在混播群 落中表现最佳的物种 (燕麦)并不是单播生产力水平 最高的物种 (苏丹草)。在混播群落中 (除苏丹草 + 多花黑麦草群落和籽粒苋 + 高丹草 + 箭 $\square$ 碗豆 + 湖 南稷子群落外), 群落生产力的主要贡献者是燕麦 ( $\mathrm{C}_{3}$ 植物), 尽管随着多样性的增加, 燕麦在群落中 的密度不断减少, 但其相对产量一直保持较高的水 平(变化于 $2.07 \sim 6.62$ 之间), 即在不同的混播群落 中, 燕麦始终占据绝对优势地位, 说明燕麦在混播群 落中具有较强的资源竞争能力和生长优势, 是互补 效应的最佳受益者, 这种生产力优势在一定程度上 抵消了群落中其它表现欠佳物种的产量损失, 从而 使群落总体上表现出显著的互补效应。

表 2 各混播群落 $\boldsymbol{D}$ 值

Table $2 \quad D$ value of each diverse communities

\begin{tabular}{|c|c|c|c|c|c|c|c|c|c|c|c|}
\hline \multirow{2}{*}{$\begin{array}{c}\text { 处理 } \\
\text { Treatment }\end{array}$} & \multirow{2}{*}{$\begin{array}{l}\text { 种数 } \\
\text { No. of } \\
\text { species }\end{array}$} & \multicolumn{9}{|c|}{$D_{i}$} & \multirow{2}{*}{$D$} \\
\hline & & $\mathrm{Y}$ & $\mathrm{D}$ & $\mathrm{H}$ & $\mathrm{S}$ & G & C & $\mathrm{J}$ & M & $\mathrm{Z}$ & \\
\hline MY & 2 & $1.03 \pm 0.06$ & & & & & & & $-0.27 \pm 0.05$ & & $0.38 \pm 0.07$ \\
\hline GD & 2 & & $-0.76 \pm 0.15$ & & & $0.91 \pm 0.19$ & & & & & $0.07 \pm 0.01$ \\
\hline YHS & 3 & $2.33 \pm 0.19$ & & $-0.57 \pm 0.07$ & $-0.47 \pm 0.10$ & & & & & & $0.43 \pm 0.04$ \\
\hline MYH & 3 & $2.23 \pm 0.21$ & & $-0.43 \pm 0.08$ & & & & & $0.45 \pm 0.04$ & & $0.7 \pm 0.14$ \\
\hline YSDH & 4 & $2.89 \pm 0.31$ & $0.31 \pm 0.03$ & $-0.55 \pm 0.09$ & $-0.85 \pm 0.17$ & & & & & & $0.4 \pm 0.06$ \\
\hline ZGJH & 4 & & & $0.92 \pm 0.18$ & & $1.63 \pm 0.18$ & & $-0.34 \pm 0.05$ & & $0.05 \pm 0.03$ & $0.5 \pm 0.03$ \\
\hline CJYMZ & 5 & $4.39 \pm 0.45$ & & & & & $-0.34 \pm 0.06$ & $-0.28 \pm 0.04$ & $-0.57 \pm 0.18$ & $-0.16 \pm 0.01$ & $0.6 \pm 0.19$ \\
\hline ZGMSC & 5 & & & & $0.44 \pm 0.07$ & $2.01 \pm 0.71$ & $-0.31 \pm 0.03$ & & $0.24 \pm 0.01$ & $-0.31 \pm 0.02$ & $0.4 \pm 0.08$ \\
\hline CMHYSZ & 6 & $4.45 \pm 0.51$ & & $-0.52 \pm 0.05$ & $-0.61 \pm 0.13$ & & $-0.13 \pm 0.03$ & & $-0.41 \pm 0.18$ & $-0.44 \pm 0.15$ & $0.39 \pm 0.06$ \\
\hline CJSHDYZ & 7 & $4.66 \pm 0.77$ & $-0.09 \pm 0.03$ & $-0.34 \pm 0.13$ & $-0.77 \pm 0.17$ & & $0.23 \pm 0.04$ & $-0.26 \pm 0.06$ & & $-0.61 \pm 0.18$ & $0.40 \pm 0.13$ \\
\hline ZCMDHGYS & 8 & $5.17 \pm 1.58$ & $-0.31 \pm 0.11$ & $-0.45 \pm 0.07$ & $-0.73 \pm 0.17$ & $-0.59 \pm 0.15$ & $0.39 \pm 0.08$ & & $-0.32 \pm 0.07$ & $-0.21 \pm 0.08$ & $0.37 \pm 0.09$ \\
\hline ZCJMDHGYS & 9 & $5.62 \pm 1.23$ & $0.02 \pm 0.08$ & $-0.24 \pm 0.06$ & $-0.77 \pm 0.09$ & $-0.48 \pm 0.06$ & $0.48 \pm 0.19$ & $-0.19 \pm 0.03$ & $-0.21 \pm 0.03$ & $-0.69 \pm 0.16$ & $0.39 \pm 0.12$ \\
\hline
\end{tabular}

Y、D、H、S、G、C、J、M、Z: 见图 1 See Fig. 1

\section{3 讨 论}

由于生态系统类型的复杂性和实验规模、实验 方法及研究者认识上的差异性, 有关物种多样性与 生态系统功能 (如生产力、养分循环、分解率及稳定 性等)关系的观察、实验和理论研究尚未得出普遍性 结论, 但大多数针对植物群落物种多样性与生产力 关系的研究都证实,在局域尺度范围内,物种多样性 对生态系统生产力存在正效应 (Hector et al ., 1999;

Tilman, 1999; Diaz \& Cabido, 2001; Loreau et al., 2001; Tilman et al., 2001; Fridley, 2003)。本研究 也进一步证实植物多样性对系统生产力有促进作 用, 但物种多样性达一定水平后 (本研究为 5 种), 物 种多样性的增加不再导致系统生产力的提高, 即群 落初级生产力并非必然地随着植物多样性的增加而 线性增加, 生产力在 $\alpha$ 多样性水平相对较低时即已 达到饱和 (Hooper, 1998), 这一点在下面的分析中
也可体现出来。同时, 在多样性-生产力关系的分析 中发现, 物种成分对生态系统生产力也有重要影响。 这可由相同的多样性水平但包含不同物种成分的群 落其生产力存在较大差别的事实得到证实 (图 2), 由 RYTs 和 $D$ 值分析也可得到证实: 相同多样性水 平的群落其互补效应却相差较大 (如毛苕子 + 燕麦 群落和多花黑麦草 + 高丹草群落、毛苕子 + 燕麦 + 湖南稷子群落和燕麦 + 湖南稷子 + 苏丹草群落)。 Hooper 和 Vitosek (1997), Tilman 等(1997)的研究也 表明, 与物种多样性相比, 物种 (或功能群)的成分对 生态系统生产力、养分循环等过程的影响更大, 说明 物种的特性是多样性研究中不可忽视的重要因素。

本研究表明,作为多样性效应的一种内在机制, 资源互补效应对群落生产力的提高有重要影响。虽 然超产效应依物种功能特性的不同变化很大，但总 体而言, 大部分混播群落中都存在超产现象。这一 结论与其它类似的研究 (Hooper \& Vitosek, 1997; 
Hooper \& Dukes，2004 )都证明资源的互补性利用是 混播群落生产力提高的主要作用机制。植物种多样 性通过生态位互补(资源瓜分) 使多样性较高的群落 能够更加充分地利用有限资源而创造出较高的生产 力 ( Tilman et al., 1997)。从理论的角度而言, 物种 间功能特征差异越大, 所组成的混播群落生产力就 越高, 但实验观察与理论预测并不完全相符。本实 验中所采用的物种 $\left(\mathrm{C}_{3}\right.$ 植物、 $\mathrm{C}_{4}$ 植物、固氮植物和双 子叶非固氮植物) 在生长特性、物候期、养分利用等 方面均存在较大差异, 按理论推测, 随着多样性的增 加, 生态位互补效应应该更加显著, 群落生产力水平 亦应不断提高。但由 4 种资源互补效应指数的计算 结果(表 1, 表 2) 和回归分析可以看出, 互补效应与 群落生产力之间并不存在正相关关系, 这说明在混 播群落(系统)中, 互补效应仅对生产力有一定的影 响, 而其它作用机制 (如竞争作用) 对生产力也有不 可忽视的影响 (Hooper, 1998)。同时, 由于所有植物 生长发育所需的资源基本相同, 在混合群落中, 物种 间资源互补性利用的机会有限, 互补作用对系统生 产力的正效应在相对较低的多样性水平下已达到饱 和(Vitousek \& Hooper, 1993), 超过该多样性水平, 生 产力不会再随多样性的增加或互补作用的存在而持 续增加。此外, 在混作群落中, 各个物种都对系统功 能产生影响, 但因各物种的功能特性差别较大, 作用 强度和作用性质有所不同, 其结果不仅会因资源的 互补性利用而使群落内部分物种的生产力提高, 同 时也会因种间的干扰性竞争而对生产力产生负效 应。因此, 由于物种成分的不同, 同一多样性水平上 的不同群落其资源互补效应和生产力水平会有较大 的变幅, 也导致了群落生产力并非必然地随着多样 性的增加而提高的现象。

在多样性-生产力关系研究中, 判定多样性效应 的潜在机制并对其进行定量描述非常必要 (Tilman et al., 1997; Hector, 1998)。本文采用了 4 种不同 的计算方法对资源互补效应进行量化描述, 不同的 方法得出的结论, 代表着不同的生态学意义。超产 效应是群落总体绝对产量的相互比较, 所反映的是 群落中功能特性存在差异的物种间相互协调、相互 竞争的结果, 是群落资源互补性利用的总体结果或 净效益。超产效应 1 的存在意味着混合群落的绝对 生产力超过其生产力水平最高组分种的单产, 是互 补效应最为严格的衡量指标, 一般较少见亦不常用 (Fridley, 2003)。相比而言, 超产效应 2 是较为温和 的指标, 应用较广。一般而言, 只要系统中出现超产
现象, 就可认为存在资源互补效应。一些研究认为, 超产效应是反映多样性增强系统功能的最佳指标 (Loreau, 1998b; Huston et al., 2000), 但也有理由认 为超产效应并非互补效应的最佳衡量标准, 其主要 的问题是超产效应可由除资源互补性利用以外的因 素(如种间个体差异或种间非补偿性作用)所致。而 相对产量的估测方法 ( $R Y T$ 和 $D$ 值) 既可对群落的 总体互补效应做出估测 ( $R Y T$ 值), 也可对其中各组 分种在资源利用上的差异做出恰当的评定 ( $R Y$ 值)。 通过 $R Y$ 值的计算, 可确切地了解群落中哪些物种 在资源的互补性利用方面存在优势, 或者说, 种间竞 争作用在多大范围内要小于种内竞争作用 ( Naeem et al. 1996), 因而不失为一种互补效应的良好测 定指标, 尽管这种方法也存在不足之处(如不能区分 种间正相互作用及生化他感作用) (Loreau, 1998b)。 类似于 $R Y T$ 指标, $D$ 值也是一个深入分析群落中各 物种具体表现的指标, 通过 $D$ 值的计算可对超产效 应的深层机理及不同物种的功能特性对互补效应的 贡献有深入的了解。该指标具有较大的伸缩性, 当 群落中所有种的 $D_{i}$ 均为正值时, 表明群落在最严格 的标准下实现超产效应; 当群落中至少有一个种的 $D_{i}$ 为正值, 其它种的 $D_{i}$ 不显著小于 0 时, 群落在较 为宽松的标准下实现超产效应 (Hooper \& Dukes, 2004）。由此可见, 资源互补效应不同的衡量指数在 反映群落超产现象时存在较大的差异, 每种计算方 法各有千秋。显而易见, 仅用一种指标衡量生态系 统多样性对生产力的影响程度, 难免失之偏颇, 因 此, 在对系统进行资源互补效应评估时, 应根据具 体情况同时采用几种不同的分析方法, 以利于对多 样性效应做出恰当的评价。

\section{参 考 文 献}

Chapin III FS, Moilanen L, Kielland K (1993). Preferential use of organic nitrogen for growth by a non-mycorrhizal arctic sedge. Nature, 361, $150-153$.

Diaz S, Cabido M (2001). Vive la difference: plant functional diversity matters to ecosystem processes. Trends in Ecology and Evolution, 16, 646-655.

Dukes JS (2001). Productivity and complementarity in grassland microcosms of varying diversity. Oikos, 94, 468-480.

Fridley JD (2001). The influence of species diversity on ecosystem productivity: how, where, and why? Oikos, 93, 514-526.

Fridley JD (2003). Diversity effects on production in different light and fertility environments: an experiment with communities of annual plants. Journal of Ecology, 91, 396-406.

Hector A (1998). The effect of diversity on productivity: detecting the role of species complementarity. Oikos, 82, 597-599.

Hector A, Schmid B, Beierkuhnlein C, Cldeira MC, Diemer M, 
Dimitrakppoulos PG, Fimm JA, Freitas H, Giller PS, Gjood J, Harris T, Högberg P, Huss-Danell K, Joshi J, Jumpponen A, Körner C, Leadley PW, Loreau M, Minns A, Mulder CPH, Donovan GO, Otway SJ, Pereira JS, Prinz A, Read DJ, Scherer-Lorenzen M, Schulze ED, Siamantziouras ASD, Spehn EM, Terry AC, Troumdis AY, Woodward FI, Yach S, Lawton JH (1999). Plant diversity and productivity experiments in European grasslands. Science, 286, $1123-1127$.

Hooper DU, Vitousek PM (1997). The effects of plant composition and diversity on ecosystem processes. Science, 277, $1302-$ 1305 .

Hooper DU (1998). The role of complementarity and competition in ecosystem responses to variation in plant diversity. Ecology, 79, $704-719$.

Hooper DU, Dukes J (2004) . Overyielding among plant functional groups in a long-term experiment. Ecology Letters, 7, 95-105.

Huston MA, Aarssen LW, Austin MP, Cade BS, Fridley JD, Garnier E, Grime JP, Larenroth WK, Thompson K, Vandermeer JH, Wardle DA (2000) . No consistent effect of plant diversity on productivity. Science, 289, 1255.

Jonssen M, Malmqvist B (2003) . Mechanisms behind positive diversity effects on ecosystem functioning: testing the facilitation and interference hypotheses. Oecologia, 134, 554- 559 .

Loreau M (1998a). Biodiversity and ecosystem functioning: a mechanistic model. Proceedings of the National Academy of Sciences of the United States of America, 95, 5632 - 5636.

Loreau M (1998b). Separating sampling and other effects in biodiversity experiments. Oikos, 82, 600-602.

Loreau M (2000). Biodiversity and ecosystem functioning: recent theoretical advances. Oikos, 91, 3-17.

Loreau M, Hector A (2001). Partitioning selection and complementarity in biodiversity experiments. Nature, 412, $72-76$.
Loreau M, Naeem S, Inchausti P, Bengtsson J, Grime JP, Hector A, Hooper DU, Huston MA, Raffaelli D, Schmid B, Tilman D, Wardle DA (2001). Biodiversity and ecosystem functioning: current knowledge and future challenges. Science, 294, 804-808.

Naeem S, Häkansson K, Lawton JH, Crawley MJ, Thompson LJ (1996). Biodiversity and plant productivity in a model assemblage of plant species. Oikos, 76, 259-264.

Symstad AJ, Tilman D, Willson J, Knops JMH ( 1998). Species loss and ecosystem functioning: effects of species identity and community composition. Oikos, 81, 389-397.

Tilman D, Wedin D, Knops J (1996) . Productivity and sustainability influenced by biodiversity in grassland ecosystems. Nature, $379,718-720$.

Tilman D, Lehman CL, Thomson KT (1997). Plant diversity and ecosystem productivity: theoretical considerations. Proceedings of the National Academy of Sciences of the United States of America, 94, $1857-1861$.

Tilman D (1999). The ecological consequences of changes in biodiversity: a search for general principles. Ecology, 80, 1455 1475 .

Tilman D (2000). Causes, consequences and ethics of biodiversity. Nature, 405, $208-211$.

Tilman D, Reich P, Knops J, Wedin D, Mielke T, Lehman C (2001). Diversity and productivity in a long-term grassland experiment. Science, 249, $843-845$.

Vitousek PM, Hooper DU (1993). Biological diversity and terrestrial ecosystem biogeochemistry. In: Schulze ED, Mooney HA eds. Biodiversity and Ecosystem Function. Springer-Verlag Press, Berlin, Germany, 3-14.

Wardle DA (1999) . Is “sampling effect” a problem for experiments investigating biodiversity-ecosystem function relationships? Oikos, $87,403-407$. 